\title{
DFB Quantum Cascade Laser Arrays
}

\section{Citation}

Lee, Benjamin G., Mikhail A. Belkin, Christian Pflügl, Laurent Diehl, Haifei A. Zhang, Ross M. Audet, Jim MacArthur, David P. Bour, Scott W. Corzine, Gloria E. Hofler, Federico Capasso. 2009. DFB quantum cascade laser arrays. IEEE Journal of Quantum Electronics 45(5): 554-565.

\section{Published Version}

doi:10.1109/JQE.2009.2013175

\section{Permanent link}

http://nrs.harvard.edu/urn-3:HUL.InstRepos:3445981

\section{Terms of Use}

This article was downloaded from Harvard University's DASH repository, and is made available under the terms and conditions applicable to Other Posted Material, as set forth at http:// nrs.harvard.edu/urn-3:HUL.InstRepos:dash.current.terms-of-use\#LAA

\section{Share Your Story}

The Harvard community has made this article openly available.

Please share how this access benefits you. Submit a story.

Accessibility 


\title{
DFB Quantum Cascade Laser Arrays
}

\author{
Benjamin G. Lee, Mikhail A. Belkin, Christian Pflügl, Laurent Diehl, Haifei A. Zhang, \\ Ross M. Audet, Jim MacArthur, David P. Bour, Fellow, IEEE, Scott W. Corzine, Gloria E. Höfler, and \\ Federico Capasso, Fellow, IEEE
}

\begin{abstract}
DFB quantum cascade laser (DFB-QCL) arrays operating between 8.7 and $9.4 \mu \mathrm{m}$ are investigated for their performance characteristics-single-mode selection of the DFB grating, and variability in threshold, slope efficiency, and output power of different lasers in the array. Single-mode selection refers to the ability to choose a desired mode/frequency of laser emission with a DFB grating. We apply a theoretical framework developed for general DFB gratings to analyze DFB-QCL arrays. We calculate how the performance characteristics of DFB-QCLs are affected by the coupling strength $\kappa L$ of the grating, and the relative position of the mirror facets at the ends of the laser cavity with respect to the grating. We discuss how single-mode selection can be improved by design. Several DFB-QCL arrays are fabricated and their performance examined. We achieve desired improvements in single-mode selection, and we observe the predicted variability in the threshold, slope efficiency, and output power of the DFB-QCLs. As a demonstration of potential applications, the DFB-QCL arrays are used to perform infrared absorption spectroscopy with fluids.
\end{abstract}

Index Terms-DFB lasers, infrared spectroscopy, mid-infrared, quantum cascade lasers (QCLs), semiconductor lasers.

\section{INTRODUCTION}

$\mathbf{M}$ ID-INFRARED quantum cascade lasers (QCLs) are unipolar semiconductor lasers that utilize resonant tunneling and optical transitions between electronic states in the conduction band of a multi-quantum-well heterostructure [1], [2]. The emitted photon energy of QCLs is determined by the thicknesses of the wells and barriers in the heterostructure and can be chosen by design using bandgap engineering. QCLs have been shown to operate reliably in the wavelength range 3-24 $\mu \mathrm{m}$ and achieve high output powers in continuous-wave

Manuscript received September 19, 2008; revised December 03, 2008. Current version published April 17, 2009. This work was supported in part by the Defense Advanced Research Projects Agency (DARPA) Optofluidics Center under Grant HR0011-04-1-0032 and in part by Massachusetts Institute of Technology (MIT) Lincoln Laboratory under Department of Defense Award FA8721-05-C-0002.

B. G. Lee, C. Pflügl, L. Diehl, H. A. Zhang, J. MacArthur, and F. Capasso are with the School of Engineering and Applied Sciences, Harvard University, Cambridge MA 02138 USA (e-mail: capasso@ seas.harvard.edu).

M. A. Belkin was with Harvard University, Cambridge, MA 02138 USA. He is now with the University of Texas at Austin, Austin, TX 78712 USA.

R. M. Audet was with Harvard University, Cambridge, MA 02138 USA. He is now with Stanford University, Stanford, CA 94305 USA.

D. P. Bour was with Agilent Laboratories, Palo Alto, CA 94304, USA. He is now with Photodigm, Inc., Richardson, TX 75081 USA, and also with BridgeLux, Inc., Sunnyvale, CA 94089 USA.

S. W. Corzine was with Agilent Laboratories, Palo Alto, CA 94304 USA. He is now with Infinera, Sunnyvale, CA 94089 USA.

G. E. Höfler was with Agilent Laboratories, Palo Alto, CA 94304 USA. He is now with Argos Technologies, Santa Clara, CA 95051 USA.

Color versions of one or more of the figures in this paper are available online at http://ieeexplore.ieee.org.

Digital Object Identifier 10.1109/JQE.2009.2013175 operation at room temperature [3], [4]. QCLs can be grown by metal-organic vapor phase epitaxy (MOVPE) [5] with quality comparable to the best devices grown by molecular beam epitaxy (MBE). Moreover, they can be designed with broadband gain, with full-width at half-maximum (FWHM) of more than $300 \mathrm{~cm}^{-1}$, enabling wide wavelength coverage for spectroscopic applications [6].

The mid-infrared spectral region is important, among other reasons, for chemical sensing and analysis. Many gas- and liquid-phase chemicals have characteristic absorption features in this region that can be used to identify them. Applications of QCLs in chemical sensing include, but are not limited to, medical diagnostics, such as breath analysis, pollution monitoring, environmental sensing of the greenhouse gases responsible for global warming, and remote detection of toxic chemicals and explosives [7].

For most applications, it is necessary to have QCL devices operating at a single desired frequency. Single-mode operation can be achieved by processing QCLs as DFB lasers [8], or by incorporating QCLs within an external cavity with a rotating diffraction grating that provides tunable, single-mode operation [9], [10]. External cavity versions are broadly tunable (over 200 $\mathrm{cm}^{-1}$ ) but complex to build, requiring careful alignment and high-quality antireflection coatings. In addition, two piezoelectric controllers are necessary to vary the cavity length and rotate the grating to ensure mode-hop free tuning. DFB QCLs are very compact (a few millimeters in length) and can easily be microfabricated in large quantities, but the tunability of a single device is limited to temperature tuning of $\sim 0.5 \mathrm{~nm} / \mathrm{K}$. Our study has been to develop a broadly tunable single-mode QCL source that combines the advantages of external cavity and DFB devices.

Our device is based on an array of DFB-QCLs, monolithically integrated on the same chip, as outlined in our previous work [11]. We fabricated several arrays of 32 lasers on a single chip, as shown in Fig. 1. Each individual laser in the array is designed to emit at a different frequency with the entire array covering a range of $90 \mathrm{~cm}^{-1}$. The spacing of the emission frequencies is sufficiently small such that for any frequency within the range covered by the array, one can select a laser in the array, tune its emission frequency by adjusting its temperature, and produce single-mode emission at the desired frequency.

In this paper, we consider the performance characteristics of these DFB-QCL arrays, including single-mode selection, threshold, slope efficiency, and output power. Single-mode selection is defined as the ability to choose a desired mode/frequency of laser emission with a DFB grating. The single-mode selection, threshold, slope efficiency, and output power are a function of the coupling strength $\kappa L$ of the DFB grating and the position and reflectivity of the end facet mirrors. 


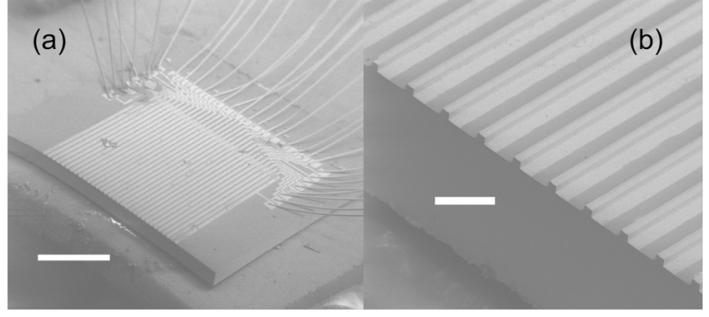

Fig. 1. (a) SEM image of the array with 32 lasers. The diagonal stripes are individual laser ridges, and there are wirebonds connected to bonding pads in the upper right portion of the image. The white bar corresponds to $1 \mathrm{~mm}$. (b) Magnified view of the laser ridges, again with the ridges running diagonally and the front facet of each laser visible. The white bar corresponds to $100 \mu \mathrm{m}$.

The structure of the paper is as follows. In Section II, the QCL structure and the design of the DFB grating structure used for the lasers in our DFB-QCL array are described. Then, in Section III, we present a theoretical analysis of the effect of coupling strength and the end facet mirrors on the properties of DFB lasers and our DFB-QCL array. The fabrication of DFB-QCL arrays is described in Section IV, and the results of testing the arrays are discussed in Section V. Finally, in Section VI, we demonstrate a potential application of the array to absorption spectroscopy.

\section{WAVEGUIDE StRUCtuRE AND DFB GRATING DESIGN}

To inform our discussion of the waveguide structure and grating design, we briefly review the properties of DFB gratings. We recall that a DFB grating has a periodic variation in refractive index that gives rise to a photonic gap around the Bragg frequency; the DFB supports lasing for longitudinal modes on either side of this gap. There are two modes that are directly at either edge of the photonic gap-these two modes have the lowest lasing threshold of all the possible modes supported by the DFB. We will call these the low- and high-frequency modes for the rest of this paper-the modes have frequencies $\nu_{1}$ and $\nu_{2}$, respectively. The low-frequency mode is more concentrated in the higher index part of the grating, with a modal effective refractive index $n_{1}=c / 2 \nu_{1} \Lambda$ where $\Lambda$ is the period of the grating. The high-frequency mode is more concentrated in the lower index part of the grating, so it has a lower modal effective refractive index $n_{2}=c / 2 \nu_{2} \Lambda$.

The grating's coupling strength is a complex number $\kappa$, whose real part is proportional to the photonic gap, and whose imaginary part corresponds to the difference in loss (or gain) of the two DFB modes across the gap. We can find $\kappa$ by simulating the modes numerically. In terms of the effective refractive indexes of the two modes directly across the photonic gap, $\kappa=\pi \Delta(n+i k) / \lambda_{0}$. Here, $\Delta(n+i k)$ is the difference in the complex effective refractive indexes of the two modes, and $\lambda_{0}$ is the Bragg wavelength.

In this paper, we typically speak of the coupling strength as $\kappa L$, which is a dimensionless quantity and includes the length of the grating $L$. As in previous work on DFB-QCLs [8], we design DFBs that are slightly overcoupled, with $\kappa L \sim 4$. Relatively short lasers are desirable since less current is required to pump shorter devices, so we aim for large $\kappa$.

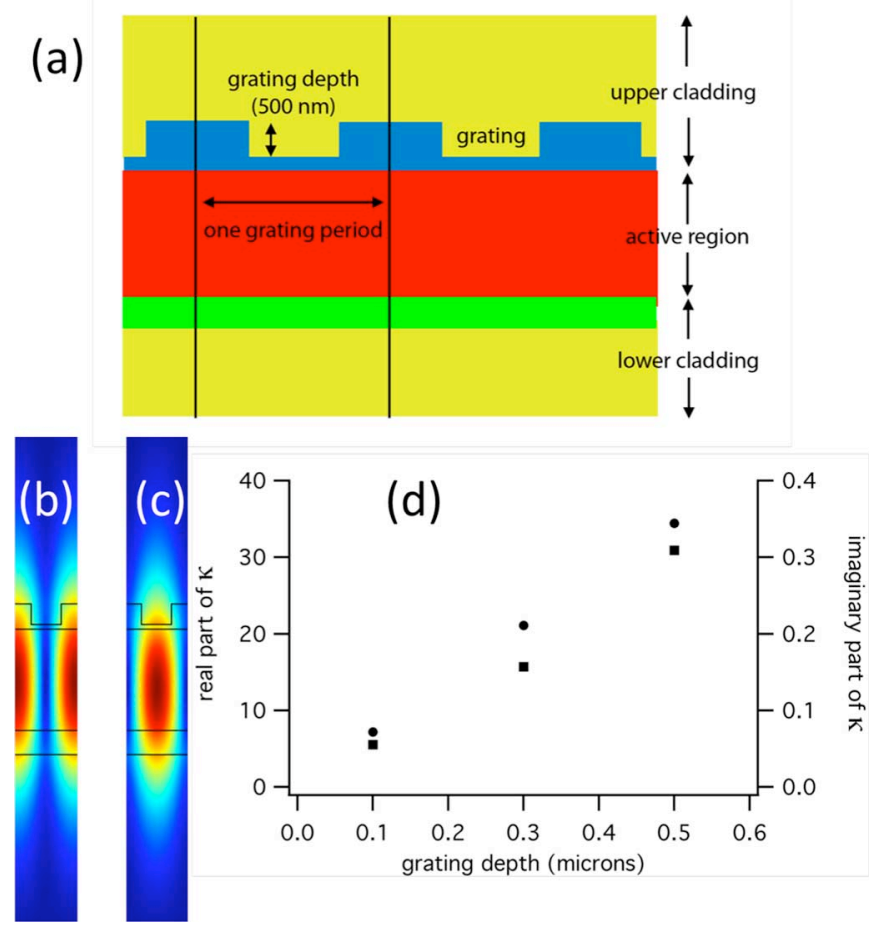

Fig. 2. (a) Schematic of the DFB-QCL waveguide structure. The grating is etched in an InGaAs layer (blue) in the top waveguide cladding, just above the active region (red). There is $\mathrm{InP}$ overgrown on top of the grating (yellow in diagram). (b) Mode simulation of the mode on the low-frequency side of the photonic gap of the DFB grating. The plot displays the magnitude of the electric field in the laser structure. The low-frequency mode has more of the electric field concentrated in the high-index part of the grating as expected. (c) Mode simulation of the mode on the high-frequency side of the photonic gap of the DFB grating. Again, we display the magnitude of the electric field. The highfrequency mode has more of the electric field concentrated in the low-index part of the grating as expected. (d) Dependence of the grating coupling strength per unit length $\kappa$ on the etch depth of the grating. The real part of $\kappa$ is shown as squares and the imaginary part as circles.

To achieve our target, we need to consider various aspects of the grating geometry, including the location of the grating, the depth of the corrugation, the duty cycle, and the choice of material for the grating. These choices influence the coupling strength of the grating, and also the optical losses of the waveguide.

Here, we choose to make a first-order DFB that is formed in the upper waveguide cladding of the QCL, as an etched corrugation in a buried InGaAs layer just above the active region of the laser [Fig. 2(a)]; this design follows a body of previous work outlined in [8]. Referring to Fig. 2(a), the DFB-QCL structure consists of a bottom waveguide cladding of $4 \mu \mathrm{m}$ of InP doped $1 \times 10^{17} \mathrm{~cm}^{-3}$ (yellow), followed by $580 \mathrm{~nm}$ of InGaAs doped $3 \times 10^{16} \mathrm{~cm}^{-3}$ (green), a 2.4- $\mu$ m-thick lattice-matched active region (red), $580 \mathrm{~nm}$ of InGaAs doped $3 \times 10^{16} \mathrm{~cm}^{-3}$ where the grating is etched $500 \mathrm{~nm}$ deep (blue), and a top waveguide cladding (yellow), consisting of $4 \mu \mathrm{m}$ of InP doped $1 \times$ $10^{17} \mathrm{~cm}^{-3}$ and $0.5 \mu \mathrm{m}$ of InP doped $5 \times 10^{18} \mathrm{~cm}^{-3}$. The active region consists of 35 stages based on a bound-to-continuum design emitting at $\sim 9 \mu \mathrm{m}[9]$.

The grating provides DFB by having a refractive index contrast between the corrugated InGaAs layer and InP material that is overgrown above it. Locating the grating just above the active region means that there is excellent overlap between the 
laser mode and the grating, resulting in a larger grating coupling strength as compared to surface gratings. The duty cycle of the grating is chosen to be $50 \%$, which is typical of first-order DFBs, as this maximizes the refractive index contrast. Finally, we performed simulations to determine the appropriate etch depth of the grating for our desired grating coupling strength.

In a 2-D simulation done using the commercial software COMSOL 3.2, we numerically find the longitudinal modes of the structure shown in Fig. 2(a), for a fixed transverse mode (i.e., $\mathrm{TM}_{00}$ ). Fig. 2(b) and (c) shows the mode profile calculated for the low- and high-frequency DFB modes, for a single period of the grating. In the grating structure, the part that is indented down ("grating troughs") has a lower effective refractive index than the part that is raised up ("grating peaks"). As previously mentioned, the low-frequency mode has higher electric field magnitude in the grating peaks that is the higher index part of the grating, and also that the high-frequency mode is more concentrated in the lower index part of the grating.

We perform the simulation for different grating depths. From the simulation, we determined the emission frequencies of the two DFB modes and also the effective refractive index experienced by each mode. For $500 \mathrm{~nm}$ grating depth, the complex effective refractive indexes $(n+i k)$ of the low- and high-frequency modes were $3.191+5.85 \times 10^{-4} i$ and $3.182+5.34 \times$ $10^{-4} i$, respectively. The real part of these numbers corresponds to the mode effective refractive index, while the imaginary part corresponds to waveguide loss/absorption $\alpha_{w}=4 \pi \nu k / c$. These waveguide losses are 8.1 and $7.4 \mathrm{~cm}^{-1}$ for the low- and high-frequency modes, which is comparable to $8.3 \mathrm{~cm}^{-1}$ found experimentally for buried heterostructure QCLs with a similar waveguide [5]. The origin of the waveguide losses is free carrier absorption due to the doping of the semiconductor layers.

Using the values of refractive index from the simulation, we calculated that the coupling strength per unit length of the 500 $\mathrm{nm}$ deep grating is $\kappa=(31+0.17 i) \mathrm{cm}^{-1}$. The grating coupling strength per unit length was also found for shallower gratings of $100 \mathrm{~nm}$ and $300 \mathrm{~nm}$ depth [Fig. 2(d)]. With shallower gratings, there is less index contrast, so $\kappa$ is smaller. Since we desire short devices for lower pump power and $\kappa L \sim 4$, we chose to etch the grating $500 \mathrm{~nm}$ deep, so that devices can be only $\sim 2 \mathrm{~mm}$ long.

\section{DFB Coupling Strength AND End FACET MirRorS}

The coupling strength of the DFB grating and the presence of mirrors have a significant impact on the performance of the device, including single-mode selection and power output. In order to gain a greater understanding of these effects, we present a theoretical analysis of the DFB coupling strength. We first show the intensity profile of the laser light along the length of the DFB for an idealized DFB without end mirrors, and then, proceed to discuss the impact of reflections from end facet mirrors. It is known that reflectivity and position of the end facet mirrors are critical factors affecting power output and single-mode selection. We investigate ways to decrease the effect of the mirrors and improve single-mode selection for DFB-QCLs.

\section{A. Intensity Profile and Power Output}

We illustrate in Fig. 3(a) the intensity profile along the length of the laser ridge for different magnitudes of the coupling constant $|\kappa L|$ in an approximation by Kogelnik and Shank [12] where we assume no mirror reflections. This figure shows that the light is more confined to the central part along the length of the cavity when $\kappa L$ is large, versus being more evenly distributed throughout the cavity when $\kappa L$ is smaller.

Taking into account the end mirror reflections in a real device, we will show that the relative position of the end facets can make a large difference in the intensity profile along the length of the cavity. Here, we build upon work by Streifer et al. [13] who treated the case of a finite-length grating with end reflectors outside the region where the grating exists. They allowed the relative position of the end reflectors with respect to the grating to vary. This matches our case, where the position of our end facet mirrors relative to the grating is basically random for each of the lasers in the array, as we have no precise control over position of the facet with our present fabrication methods.

Following the work of Streifer et al. [13], we consider coupled-wave solutions of the electric field along the grating. The grating couples right- and left-traveling waves $R(z)=r_{1} e^{\gamma z}+$ $r_{2} e^{-\gamma z}$ and $S(z)=s_{1} e^{\gamma z}+s_{2} e^{-\gamma z}$ where $r_{1}, r_{2}, s_{1}, s_{2}$ are constants to be determined and $\gamma$ obeys the eigenvalue equation

$$
\begin{aligned}
(\gamma L)^{2} \Phi & +(\kappa L)^{2} \sinh ^{2}(\gamma L)\left(1-\rho_{l}^{2}\right)\left(1-\rho_{r}^{2}\right) \\
& +2 i \kappa L\left(\rho_{l}+\rho_{r}\right)\left(1-\rho_{l} \rho_{r}\right) \gamma L \sinh (\gamma L) \cosh (\gamma L)=0 .
\end{aligned}
$$

Also, $\gamma$ satisfies the dispersion relation

$$
\begin{aligned}
\gamma^{2} & =(\alpha-i \delta)^{2}+\kappa^{2} \\
\Phi & =\left(1+\rho_{l} \rho_{r}\right)^{2}-4 \rho_{l} \rho_{r} \cosh ^{2}(\gamma L) .
\end{aligned}
$$

Here, $\rho_{l}$ and $\rho_{r}$ are the reflectivities of the left and right mirrors, respectively, including both the amplitude and phase of the reflection. $\alpha$ is the total loss of the mode, which can also be seen as the threshold gain required for that mode to lase. $\delta$ is the frequency of the mode relative to the Bragg frequency; in particular, the low- and high-frequency modes directly on either side of the bandgap have $\delta<0$ and $\delta>0$, respectively.

If we set $\rho_{l}=\rho_{r}=0$, we recover the specific case of no mirror reflections (Kogelnik and Shank [12])

$$
(\gamma L)^{2}+(\kappa L)^{2} \sinh ^{2}(\gamma L)=0
$$

By solving the general eigenvalue equation, we can find the allowed values for $\gamma$, corresponding to different longitudinal modes supported by the grating. The solutions also yield the total loss $\alpha$ and frequencies $\delta$ (relative to the Bragg frequency) of these modes. Moreover, the intensity profile of each mode along the length of the cavity can be obtained from the sum of the right- and left-traveling waves $R(z)$ and $S(z)$. We investigate a few illustrative cases to predict the performance of DFB-QCL arrays.

If the mirrors are arranged symmetrically (i.e., the relative position of both end facets with respect to the grating grooves 

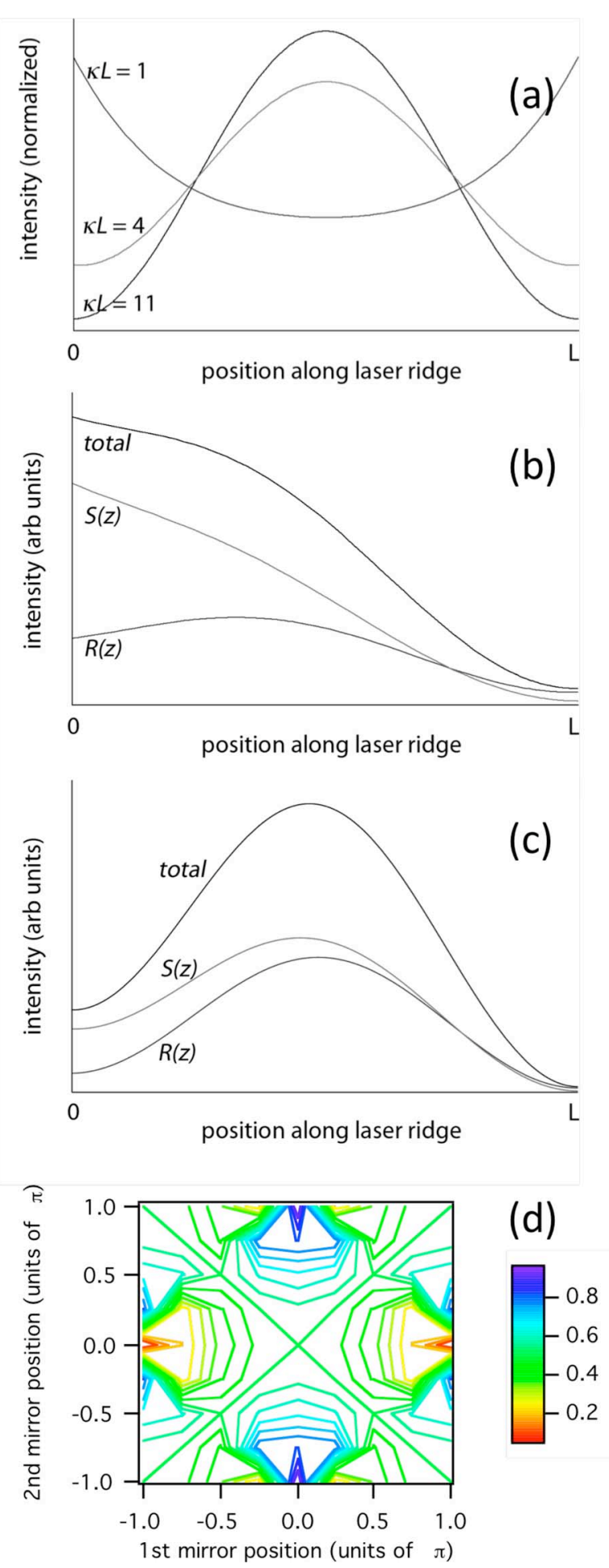

Fig. 3. (a) Intensity profile of laser light along the length of the cavity, for coupling constants $\kappa L=1,4,11$ in the absence of mirrors. (b) Intensity profile for $\kappa L=4$ and a very asymmetric mirror configuration-one mirror is coincident with the grating peaks and the other with the grating troughs. (c) Intensity profile for $\kappa L=11$ and a very asymmetric mirror configuration-one mirror is coincident with the grating peaks and the other with the grating troughs. (d) Plot of the power emitted from the front facet of the laser, as a fraction of the total power from both facets, for arbitrary positions of the end facet mirrors relative to the grating. The grating coupling strength here is $\kappa L=4.6+0.025 i$.

is the same), then, clearly, the intensity profile remains symmetric. However, in general, the mirrors are not arranged sym- metrically. In Fig. 3(b) and (c), we illustrate particularly asymmetric cases, where one mirror is coincident with a "trough" of the grating while the other is coincident with a "peak" of the grating.

In Fig. 3(b), we see an asymmetric intensity profile for a grating where $\kappa L=4$ and the mirror reflectivity is $30 \%$. The curves in the graph are the total intensity, and the intensities of the right- and left-traveling waves $R(z)$ and $S(z)$. The power output from the right facet is given by the intensity of the righttraveling wave at the facet multiplied by the transmission coefficient of that facet (70\%). Similarly, the power output from the left facet is the intensity of the left-traveling wave at the facet multiplied by the transmission coefficient of that facet. From the large asymmetry of the intensity profile, we can see that the light intensity output from one facet can be an order of magnitude greater than from the other facet. Fig. 3(c) shows that the asymmetry of the light output remains for larger $\kappa L$-here, we show the case where $\kappa L=11$. The overall intensity profile varies less, since most of the mode is concentrated in the center of the laser cavity. However, the ratio between the intensities of light output from the left and right facets remains large.

We repeat this calculation for arbitrary mirror positions and a fixed $\kappa L(=4.6+0.025 i)$. Fig. 3(d) shows the power output from the front facet of the laser, as a fraction of the total power emitted from both end facets. While a slight majority of plotted points have between $40 \%$ and $60 \%$ of the light being emitted from the front facet, there are mirror configurations that give 5\% or $95 \%$ of the power from one facet. Thus, there can be a large variability in the output light intensity measured from the front facet of lasers with different mirror configurations, as great as an order of magnitude in range. With an array of DFB-QCLs where all the end mirrors have arbitrary positions relative to the grating, this variability will be seen in large differences in the output power measured from different lasers in the array. This variability will also appear in the slope efficiencies $d P / d I$ of different lasers in our DFB-QCL arrays.

Our calculations demonstrate the inherently large variability in the light intensity output of DFB-QCLs in an array when we have significant reflectivity from the end mirrors and arbitrary mirror positions. In order to remove this variability, one could either find a way to reliably terminate the laser cavity at the same point relative to the local grating for each of the lasers in the array, or reduce the reflectivity of both mirror facets with antireflection coatings.

\section{B. Single-Mode Selection}

The end facet mirrors can also have a significant impact on the single-mode selection, which we defined earlier as the ability to choose a specific desired mode/frequency of laser emission with a DFB grating. More specifically, we will have good singlemode selection if we can reliably cause only the low-frequency (or only the high-frequency) mode to lase for every DFB-QCL in the array.

A mode lases at a specific frequency when the gain at this frequency exactly compensates for the total optical losses, which include the waveguide losses and the mirror losses over one round-trip in the cavity. For our lasers, we can calculate from the gain spectrum (FWHM of $300 \mathrm{~cm}^{-1}$ ) that the amount of modal 
gain for lasing at closely spaced frequencies (i.e., $3.1 \mathrm{~cm}^{-1}$ apart) differs by $1 \%$ or less. This means that the difference in total loss between the two DFB modes primarily determines which one of them actually lases. Specifically, the DFB mode with the lower optical loss will lase.

The two DFB modes have different waveguide losses. From the previous calculations of waveguide losses, these are 8.1 and $7.4 \mathrm{~cm}^{-1}$ for the low- and high-frequency DFB modes, respectively. Now, if the effect of end facet mirrors could be ignored, then the mode with the smaller waveguide losses will always lase. However, the presence of end facet mirrors gives reflections that constructively or destructively interfere with the DFB modes in the laser cavity. This interference affects the mirror loss of each mode, and can determine which mode lases.

We note that the effect of the mirrors is largest when the position of both mirrors coincide with a peak in electric-field amplitude of one DFB mode, which is also when the mirrors are at a node for the other DFB mode. When both mirrors coincide with the peaks, then the reflections from the end mirrors maximally constructively interfere with the mode present in the laser cavity. This results in a lower total loss, due to the constructive contribution of the mirrors. When both mirrors coincide with the nodes, then the reflections from the end mirrors destructively interfere with the mode present in the laser cavity. This results in a higher total loss, due to the contribution of the mirrors.

Using the method developed by Streifer et al. [13], we calculated the total losses for the two DFB modes for arbitrary end mirror positions and plotted the difference between these losses in Fig. 4. The total losses include both the waveguide losses and the mirror losses, which can vary by several inverse centimeter, and thus dominate the difference in waveguide losses. Here, the possible positions of the mirrors with respect to the grating are designated by a phase, which can range between 0 and $\pi$-corresponding to the case where the mirror is either exactly in line with a grating trough or with a grating peak. A total loss difference above zero means that the high-frequency mode has greater loss than the low-frequency mode. When the plot is below zero, then the high-frequency mode has the lower loss.

Fig. 4(a) shows the case for a grating with coupling strength $\kappa L=4.6+0.025 i$ and both mirrors having $30 \%$ reflectivity. This corresponds to a laser array that is $1.5 \mathrm{~mm}$ long, fabricated with the grating structure we previously simulated. Fig. 4(a) predicts that, on average with random mirror positions, the highfrequency mode will have lower loss $57 \%$ of the time, while the low-frequency mode will have lower loss $43 \%$ of the time. This means that, for a sufficiently large array of lasers where the statistics hold, $57 \%$ of the time the high-frequency mode will lase and $43 \%$ of the time the low-frequency one will.

One way of decreasing the effect of the end mirror facets is to put an antireflection coating on the output facet, which will decrease the reflectivity of that facet, and hence its effect on the total losses of the DFB modes. In Fig. 4(b), we show the case where we have a grating with coupling strength $\kappa L=$ $4.6+0.025 i$, the first mirror having an antireflection coating that reduces its reflectivity to $1 \%$ and the second mirror having $30 \%$ reflectivity. We see that the first mirror now barely has an effect on the losses, which increases the probability that the high-frequency mode lases to $75 \%$, while now the low-frequency mode
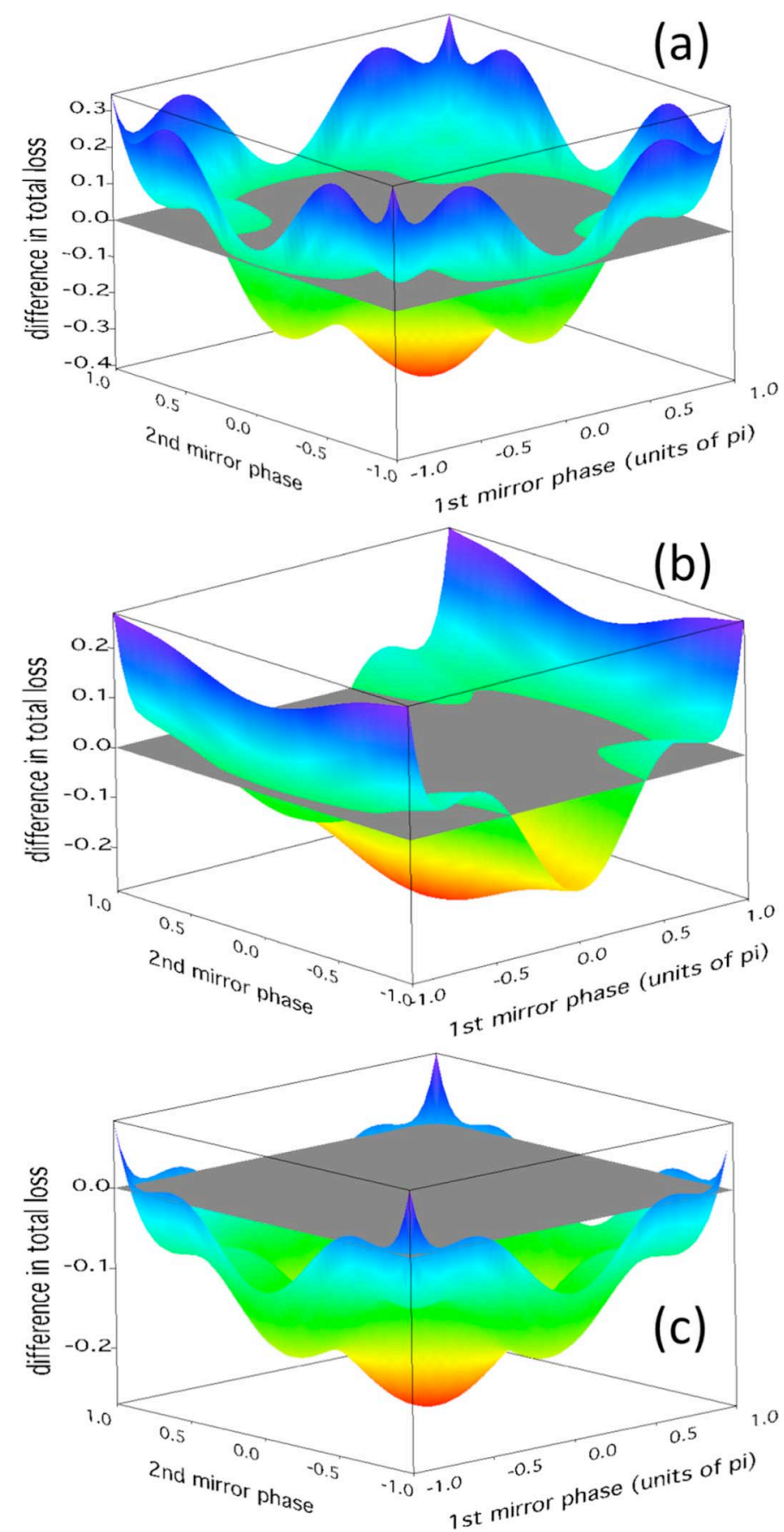

Fig. 4. Three plots showing the difference in total loss between the high- and low-frequency modes, for different coupling strengths $\kappa L$ of the grating and different configurations of the end mirrors. The possible positions of the mirrors with respect to the grating are designated by a phase, which can range between 0 and $\pi$-corresponding to the case where the mirror is exactly in line with a grating groove and exactly antialigned, respectively. When the surface of the plot of total loss is above zero, it means that the high-frequency mode has greater loss than the low-frequency mode. When the plot is below zero, then the highfrequency mode has the lower loss. (a) Plot for the case where $\kappa L=4.6+$ $0.025 i$ and both mirrors have $30 \%$ reflectivity. (b) Plot for the case where $\kappa L=$ $4.6+0.025 i$, the first mirror has an antireflection coating with $1 \%$ residual reflectivity, and the second mirror has $30 \%$ reflectivity. (c) Plot for the case where $\kappa L=11+0.058 i$ and both mirrors have $30 \%$ reflectivity.

only lases $25 \%$ of the time. This is due to the smaller effect of the mirrors and the lower waveguide losses of the high-frequency mode. 
Another way to decrease the effect of the end mirrors is to have a stronger DFB grating. Having a stronger DFB grating means that more of the laser light will be reflected by the grating grooves and the laser mode will be more confined toward the center part of the laser cavity, and have much lower intensity toward the ends of the cavity where the mirrors are. This situation was illustrated in Fig. 3, and described in our previous discussion of the intensity profile of a DFB with $\kappa L=11$.

In Fig. 4(c), we show the case where $\kappa L=11+0.058 i$ and both mirrors have $30 \%$ reflectivity. This corresponds to a laser array that is $3.5 \mathrm{~mm}$ long, fabricated with the grating structure we previously simulated. Now, the loss difference due to the end mirrors for the two modes is almost always less than the difference in their waveguide losses. Hence, we are sufficiently insensitive to the end mirrors that the mode with the smaller waveguide losses, the high-frequency mode, will lase $95 \%$ of the time.

\section{DEVICE FABRICATION}

We fabricated several DFB-QCL arrays to experimentally determine their characteristics. The QCL material used to fabricate the laser arrays was grown by MOVPE. The layer structure was described in Section II.

Device processing started with the fabrication of arrays of 32 buried DFB gratings in the QCL material. Grating periods ranged between 1.365 and $1.484 \mu \mathrm{m}$, satisfying the Bragg condition for lasing wavelengths between 8.71 and $9.47 \mu \mathrm{m}$, assuming an effective refractive index of 3.19 as calculated in our mode simulations. To fabricate the buried gratings, the top waveguide cladding was removed down to the first InGaAs layer, using concentrated $\mathrm{HCl}$ as a selective wet etch. Then, a 200-nm-thick layer of $\mathrm{Si}_{3} \mathrm{~N}_{4}$ was deposited on top of the In$\mathrm{GaAs}$ by chemical vapor deposition. First-order Bragg gratings were exposed onto AZ-5214 image-reversal photoresist by optical lithography, using a photomask where the grating patterns had been defined by electron-beam writing. This pattern was transferred into the $\mathrm{Si}_{3} \mathrm{~N}_{4}$ by using a $\mathrm{CF}_{4}$-based dry etch. The gratings were then etched $500 \mathrm{~nm}$ deep into the top InGaAs layer with an $\mathrm{HBr} / \mathrm{BCl}_{3} / \mathrm{Ar} / \mathrm{CH}_{4}$ plasma in an inductively coupled plasma reactive ion etching (ICP-RIE) machine. The InP top cladding was regrown over the gratings using MOVPE.

Laser ridges, $15 \mu \mathrm{m}$ wide and spaced $75 \mu \mathrm{m}$ apart, were defined on top of the buried gratings by dry etching the surrounding areas $9 \mu \mathrm{m}$ deep with an $\mathrm{HBr} / \mathrm{BCl}_{3} / \mathrm{Ar} / \mathrm{CH}_{4}$ plasma using ICP-RIE. During this step, the back facet of the lasers was also defined. The bottom and the sidewalls of the laser ridges were insulated by $\mathrm{Si}_{3} \mathrm{~N}_{4}$, and a 400-nm-thick gold top contact was deposited. The samples were then thinned to $200 \mu \mathrm{m}$ and a metal bottom contact was deposited. Finally, the front facets of the lasers were defined by cleaving. In this paper, we discuss results from two different arrays; one of these was cleaved to obtain 1.5-mm-long lasers, and the other was cleaved to obtain 3.5-mm-long lasers. Each laser array was indium-soldered onto a copper block for testing. The entirety of each DFB laser array chip is only $4 \mathrm{~mm} \times 5 \mathrm{~mm}$ in size (Fig. 1).

\section{ARray Results}

To test an array of DFB-QCLs, we applied electrical current pulses to individual laser ridges at room temperature. We also determined the coupling strength of the grating by measuring the luminescence of the device below the lasing threshold. The luminescence spectrum was measured with a subthreshold dc current at $77 \mathrm{~K}$. The spectrum shows a photonic bandgap of 3.1 $\mathrm{cm}^{-1}$ [Fig. 5(a), inset], from which the real part of $\kappa$ is obtained $\left(\kappa=30 \mathrm{~cm}^{-1}\right)$ in excellent agreement with the theoretical prediction $\left(31 \mathrm{~cm}^{-1}\right)$.

\section{A. Short Array}

We first present the results of testing the laser array that is $1.5 \mathrm{~mm}$ long, which corresponds to a coupling strength $\kappa L=$ $4.6+0.025 i$. The spectra for the 32 lasers of this array are presented in Fig. 5(a). All the lasers operate single-mode with greater than $20 \mathrm{~dB}$ suppression of side-modes [Fig. 5(b)]. However, the emission frequencies are not spaced regularly apart (by $\sim 2.7 \mathrm{~cm}^{-1}$ ) as desired. Instead, there is an uneven pattern.

As discussed in Sections II and III, there are two possible lasing modes, which exist on either side of the photonic bandgap - the low- and high-frequency DFB modes. The uneven pattern of spectra results because some lasers are lasing in the low-frequency DFB mode while others are lasing in the high-frequency DFB mode. The frequency spacing between adjacent lasers falls under three categories: the spacing is the desired spacing $\left(2.7 \mathrm{~cm}^{-1}\right)$, the spacing is the sum of the desired spacing and the bandgap $\left(2.7+3.1=5.8 \mathrm{~cm}^{-1}\right)$, or the spacing is the difference of the desired spacing and the bandgap $\left(2.7-3.1=-0.4 \mathrm{~cm}^{-1}\right)$. These correspond, respectively, to the cases where two adjacent lasers lase on the same side of the bandgap, the first laser lases in the low-frequency mode and the second in the high-frequency mode, the first laser lases in the high-frequency mode and the second in the low-frequency mode.

This effect arises because of the impact of the end facets at either end of the laser ridges - these end facets are partially reflecting ( $\sim 30 \%$ reflectivity) mirrors. Moreover, the position of these end facets with respect to the grating can vary in a basically random manner, from the random position of the cleaved facet relative to the grating grooves. These mirror positions affect which of the two modes on either side of the photonic gap has lower total loss, and hence which mode lases. In the previous discussion of the impact of end facet mirrors and Fig. 4(a), we predicted that $57 \%$ of the time the high-frequency mode will lase, while $43 \%$ of the time the low-frequency one will. The results agree, with 18 out of 32 lasers lasing on the high-frequency mode and 14 lasers lasing on the low-frequency mode-this is $56 \%$ and $44 \%$, respectively.

We note that the mode selection is stable-the same mode lases if the laser is turned off and on again, or if the pump current is varied. This is additional evidence that the variation in mode selection is due to a fixed factor such as the mirror positions.

We also observe that there is significant variability in the slope efficiencies and peak output power of the lasers. This can be seen from the light output data in Fig. 5(c). The $I-V$ characteristic of different lasers in the array is basically identical, but 

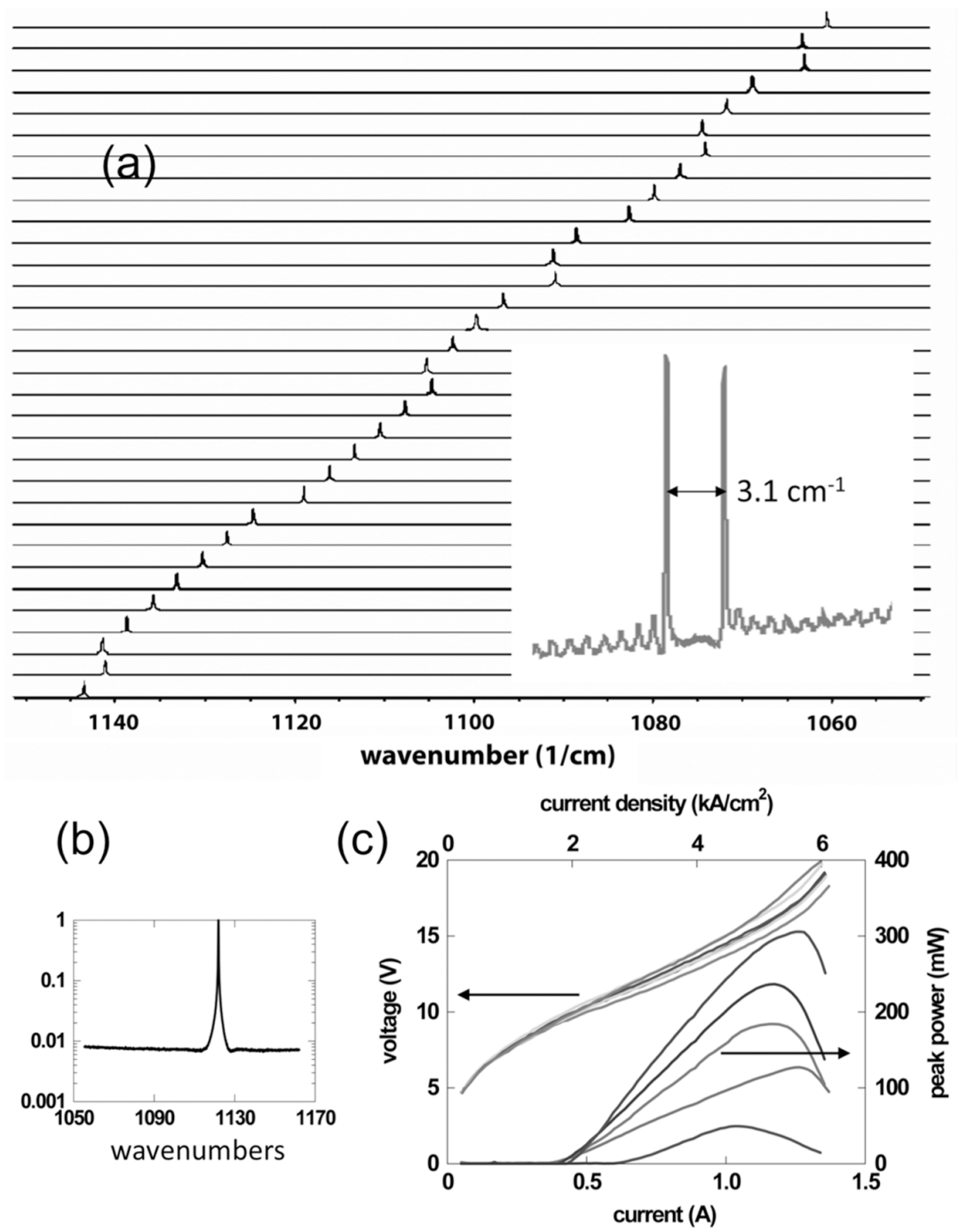

Fig. 5. (a) Spectra of 32 lasers from an array with $\kappa L=4.6+0.025 i$. All the lasers are single-mode, but 18 of the lasers are lasing on the high-frequency side of the DFB grating's photonic gap, while 14 are lasing on the low-frequency side. The inset shows the two modes on either side of the photonic bandgap. (b) Plot of the voltage (left axis) and light output intensity (right axis) from several lasers in the same array, as a function of pump current. There is a small amount of variability in the threshold current, and a larger variation in the slope efficiencies, which leads to a significant variation in the peak output power.

the light output varies by an order of magnitude, which is consistent with the discussion in Section III and Fig. 3(d).

\section{B. Array With Antireflection Coating}

We coated the front facet of the array with 1.5-mm-long lasers to try to improve the single-mode selection-specifically to increase the probability of observing the high-frequency DFB mode for each laser in the array. For the antireflection coating, we evaporated a two-layer stack of $\mathrm{YF}_{3}$ and $\mathrm{ZnSe}$, a techique also employed in [6]. $\mathrm{YF}_{3}$ has a refractive index of 1.335 at $9 \mu \mathrm{m}$ wavelength and $\mathrm{ZnSe}$ has a refractive index of 2.412. We deposited $610 \mathrm{~nm}$ of $\mathrm{YF}_{3}$ and $340 \mathrm{~nm}$ of $\mathrm{ZnSe}$ films on the front facet of the lasers-this decreases the residual reflectivity of the facet to below $1 \%$.

After applying the antireflection coating to the array, the laser characteristics were measured again. Fig. 6(a) shows the spectra of the 32 lasers in the array. Now, 25 out of 32 lasers are lasing on the high-frequency DFB mode and 6 are lasing on the low-frequency mode, with 1 laser lasing on both modes. Disregarding the one multimode laser, this means that $81 \%$ of the time the high-frequency mode lases and $19 \%$ of the time the low-frequency mode does. This is in reasonable agreement with the prediction of a 75\%/25\% split, as shown in Fig. 4(b).

Since the antireflection coating increases the mirror losses, the threshold current for lasing is slightly increased. This can be seen in Fig. 6(b). However, the slope efficiency of the lasers is also higher, since more light is coupled out of the laser cavity from the coated end. Overall, the peak output power from the lasers in the array is similar to the situation before the antireflection coating was applied. This is simply a coincidence of the fact that the effects of the increased threshold and slope efficiency affect the peak output power in opposing ways and roughly cancel each other.

Having antireflection coatings on the back facet as well would further improve the single-mode selection. However, this is difficult to realize with the present DFB-QCL array geometry where the back facets are etched. 


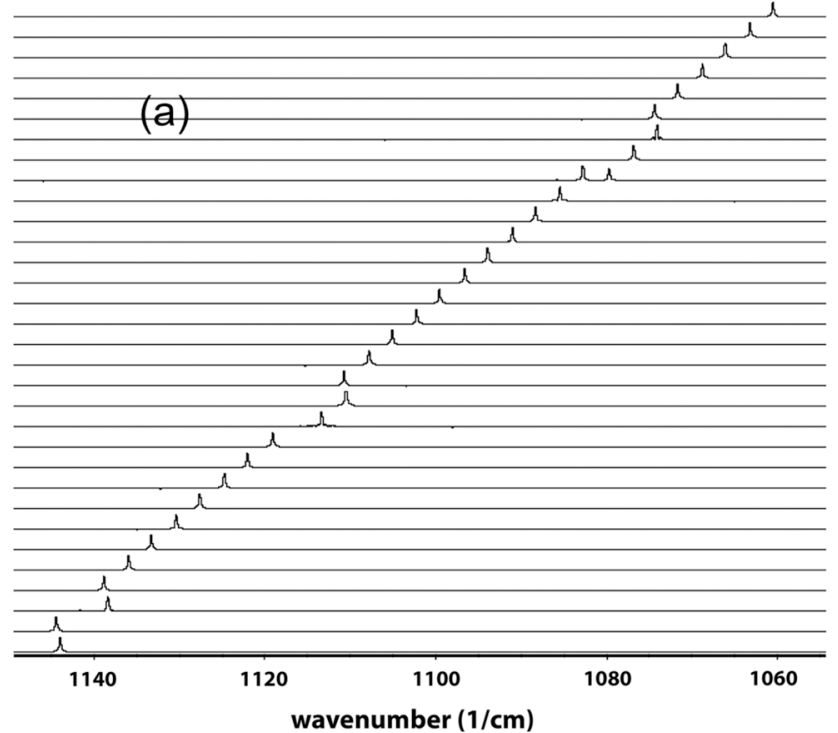

(b)

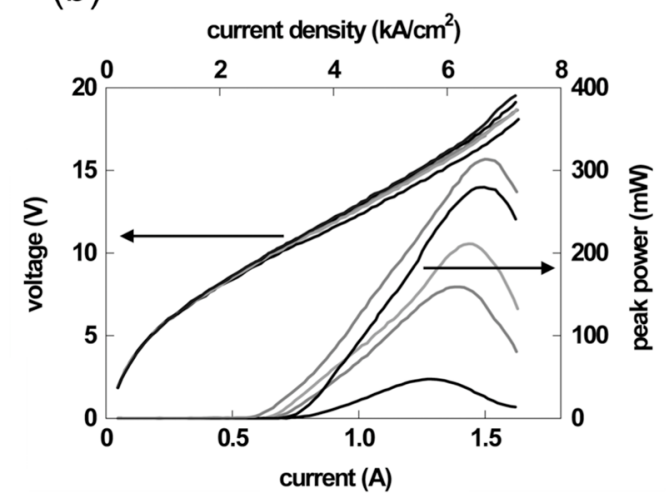

Fig. 6. (a) Spectra of 32 lasers from the array with $\kappa L=4.6+0.025 i$, after an antireflection coating is applied on the front facet, with $1 \%$ residual reflectivity. All but one of the lasers are single-mode, with 25 of the lasers are lasing on the high-frequency side of the DFB grating's photonic gap, and 6 lasing on the low-frequency side. One of the lasers has both modes lasing. (b) Plot of the voltage (left axis) and light output intensity (right axis) from several lasers in the same array, as a function of pump current.

\section{Longer Array}

We recall that an even more effective way of getting more of the lasers in an array to emit in the same DFB mode (specifically the high-frequency DFB mode) is to have an array with a stronger coupling $\kappa L$. We can achieve this by fabricating an array with longer lasers. Here, we present the results of a longer array where the lasers are $3.5 \mathrm{~mm}$ long, corresponding to $\kappa L=$ $11+0.058 i$.

Looking at the spectra of the array in Fig. 7(a), we see that all the lasers are single-mode and they all lase on the high-frequency side of the DFB grating's photonic gap. This is even better than the theoretical prediction of a $95 \% / 5 \%$ split between high- and low-frequency modes in Fig. 4(c). So, having a strongly overcoupled grating is a very effective way of suppressing the effect of the end facet mirrors in mode selection.

However, the light output intensity of the lasers suffer. Fig. 7(b) shows that the slope efficiency and the peak output power of the lasers are lower than with the shorter array. This

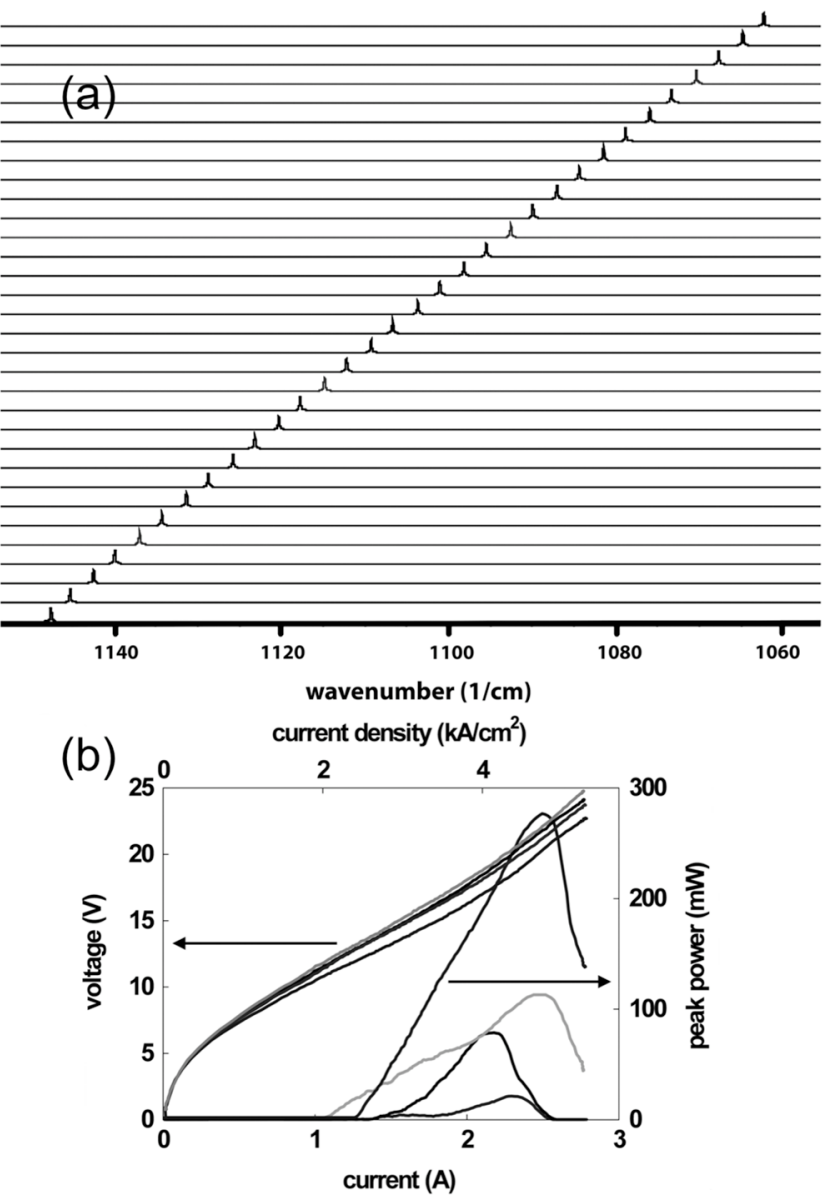

Fig. 7. (a) Spectra of 32 lasers from an array with $\kappa L=11+0.058 i$. All the lasers are single-mode, and they all lase on the high-frequency side of the DFB grating's photonic gap. (b) Plot of the voltage (left axis) and light output intensity (right axis) from several lasers in the same array, as a function of pump current.

is because the light is more highly confined in the central part along the length of the laser ridge by a larger number of reflections from the stronger grating. Less of the light makes it out of the laser cavity, so the light output is smaller. One benefit is that the threshold current density required for lasing is also smaller due to the strong optical feedback in the grating.

We see that a more strongly coupled grating can help to reliably select one of the two DFB modes to lase. However, this comes at the cost of decreasing the output power of the lasers.

\section{Threshold and Slope Efficiencies}

While we have already examined the thresholds and slope efficiencies of the laser arrays, it is worthwhile to summarize these results and compare them. One way of getting a more quantitative view, given the variability inherent in each array, is to view the thresholds and slope efficiencies as histograms. A histogram of threshold currents (or slope efficiencies) shows how many lasers in each array have thresholds (slope efficiencies) within a given range of values.

Fig. 8(a) shows a histogram of the threshold current densities for the three cases (short array, array with anti-reflection coating, longer array). The histogram confirms that the threshold current density is lowest for the longer array and 

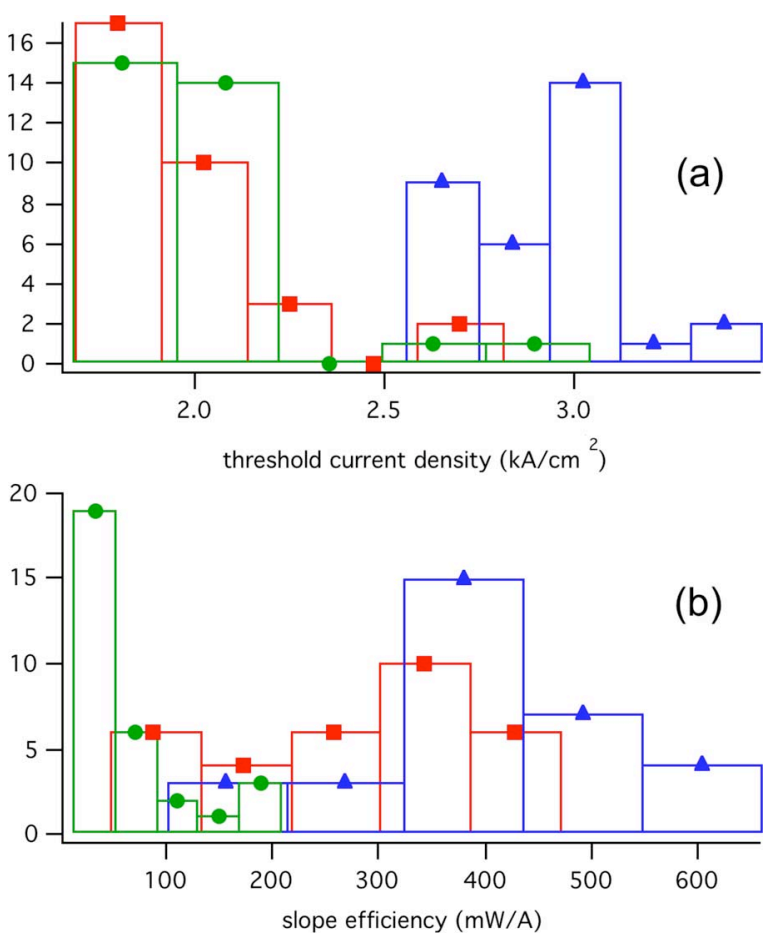

Fig. 8. (a) Histogram of the threshold current densities for the three different DFB-QCL arrays. The array with $\kappa L=4.6+0.025 i$ is denoted with square markers, and after coating the front facet with an antireflection coating, the new thresholds are denoted with triangles, and finally, the array with $\kappa L=11+$ $0.058 i$ is denoted with circles. (b) Histogram of the slope efficiencies for the three different DFB-QCL arrays. The array with $\kappa L=4.6+0.025 i$ is denoted with squares, and after coating the front facet with an antireflection coating, the new slope efficiencies are denoted with triangles, and finally, the array with $\kappa L=11+0.058 i$ is denoted with circles.

highest for the array with antireflection coating. As previously discussed, this is expected because the longer array has greater optical feedback from its grating so that the threshold should be lower; also, the antireflection coating reduces the optical feedback, giving a higher lasing threshold.

Fig. 8(b) shows a histogram of the slope efficiencies for the three cases. The histogram displays a clear trend in the magnitude of the slope efficiencies - the longer array has the lowest slope efficiencies, while the short array has higher slope efficiencies, and the antireflection-coated array has the highest. Again, this is in line with our previous discussion.

The longer array has stronger coupling $|\kappa L| \sim 11$. Thus, the light is more highly confined in the central part along the length of the laser ridge by a larger number of reflections from the stronger grating. Less of the light makes it out of the laser cavity, so the light output is smaller. The array with antireflection coating allows more light to come out of the coated end, so its slope efficiency is higher.

The histogram also shows that there is almost an order of magnitude variation in the slope efficiency for each of the three cases. This is due to the effect of the end facet mirrors. The reflections from the end facet mirrors cause highly asymmetric intensity profiles along the length of the laser cavity. This was predicted from theory in Section III. Here, we reiterate that a highly asymmetric intensity profile along the laser cavity means that the amount of light emitted from one facet of the laser does

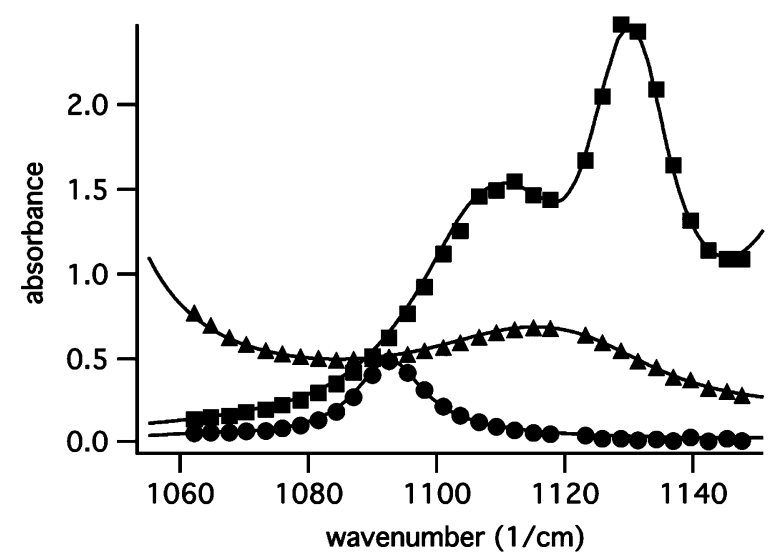

Fig. 9. Absorption spectra of isopropanol (squares), methanol (triangles), and acetone (circles) taken using the DFB-QCL array source and using a Bruker Vertex 80v FTIR (continuous lines). This was previously reported in [11].

not have to be equal to the amount emitted from the other facet. For an array of lasers, this results in a large variability in the measured output power from the front facet between different lasers in the array. As the slope efficiency is $d P / d I$, it also shows up as a large variability in the slope efficiency.

\section{APPLiCATIONS OF DFB-QCL ARRAYS}

The DFB-QCL array is a mid-infrared source that can emit any frequency within a designed range. It covers that range by being continuously tunable, since the separation in nominal emission frequencies is small enough that we can use temperature tuning to span the spacing. With such an array, we can perform infrared spectroscopy, with many potential applications in chemical sensing, including medical diagnostics such as breath analysis, pollution monitoring, and remote detection of toxic chemicals and explosives.

Here, we demonstrate the usefulness of arrays of DFB-QCLs for absorption spectroscopy with fluids. Condensed phase materials such as fluids can have significant absorption, and typically have broadened absorption features that are well suited to measurements using a wide-coverage DFB-QCL array.

We perform absorption spectroscopy by firing the array of lasers one-by-one through an analyte, and looking at the transmitted signal intensity, as compared to a reference case without the analyte. The absorption at any frequency can then be calculated by comparing the two measurements. The 3.5-mm-long DFB-QCL array was used, since all of its lasers operated singlemode at the designed frequencies.

Fig. 9 shows absorption spectroscopy performed on three fluids-isopropanol, acetone, and methanol. The analytes were contained in a transparent $\mathrm{BaF}_{2}$ fluid cell with a $23.6-\mu \mathrm{m}$ chamber thickness. The measurement result was previously reported in [11]. The absorption spectra obtained using the DFB-QCL array (only 31 lasers were used from the array since one was damaged) are shown, and compared to those obtained using a conventional Fourier transform infrared spectrometer (FTIR). The spectra took less than $10 \mathrm{~s}$ to obtain using the DFB-QCL array. The present limitation on speed is due to the fastest repetition rate $(100 \mathrm{kHz})$ achievable using electronics we custom-built for the array, and also due to the delay in 


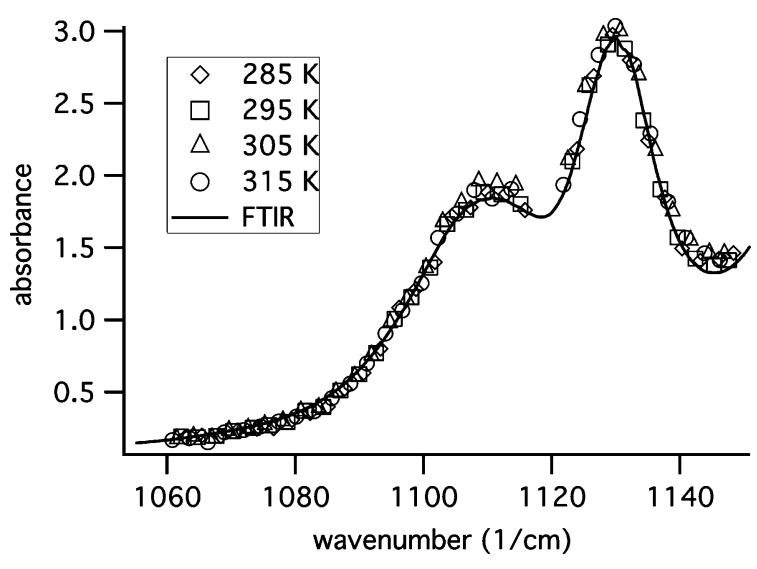

Fig. 10. Absorption spectrum of isopropanol taken using the DFB-QCL array source operated at different temperatures, in order to have a "continuous" measure of the spectrum (points). Data taken using a Bruker Vertex 80v FTIR are shown for comparison (solid line).

transmitting both control instructions and data over a slow serial connection between the electronics and our laboratory computer. With faster repetition rates and a higher data rate connection, sampling can occur much quicker and the measurement time could be reduced to milliseconds.

In order to obtain continuous spectral coverage between the nominal emission frequencies of the individual lasers in the array, one can tune the lasers in a small range. This is done by temperature tuning - the lasers can either be heated locally by applying a subthreshold dc current to tune an individual laser, or the lasers can be heated globally by changing the temperature of the heat sink on which the laser array chip sits. With dc bias current tuning, we tuned by $5 \mathrm{~cm}^{-1}$ with $300 \mathrm{~mA}$. Alternatively, by varying the heat sink temperature with a thermoelectric cooler from 252 to $325 \mathrm{~K}$, we also tuned $5 \mathrm{~cm}^{-1}$. Local heating using dc current can achieve the desired temperature in milliseconds, while heat sink temperature changes typically take tens of seconds.

Absorption spectroscopy using the DFB-QCL array with continuous coverage between the nominal emission frequencies of the individual lasers can then be performed. This is demonstrated in Fig. 10 and is reported here for the first time. Here, the temperature of the laser array chip was adjusted globally by controlling the heat sink temperature using a thermoelectric cooler. The absorption spectra obtained with the DFB-QCL array in each case agree well with the results found using FTIR.

A significant advantage of DFB-QCLs is that the brightness of the source is much greater than the thermal sources used in FTIR; thus, there can be greatly improved signal-to-noise in measurements, particularly when using condensed-phase analytes such as fluids with high absorption. Additionally, we note that the frequency resolution of our lasers is determined by their linewidths, which are $\sim 0.1 \mathrm{~nm}$ in pulsed operation and can be $<0.001 \mathrm{~nm}$ in continuous-wave operation [14]. This is significantly better than the resolution offered by a typical "bench top" FTIR $(\sim 1 \mathrm{~nm})$. Despite the narrower spectral measurement range compared to FTIR spectrometers, we believe that a spectrometer based on our DFB-QCL can provide a portable alternative to FTIR spectrometers in the mid-infrared.

\section{SUMMARY AND FUTURE DiRECTIONS}

We investigated DFB-QCL arrays for their performance characteristics-single-mode selection, threshold, slope efficiency, and output power. We observed that the single-mode selection of the DFB gratings is affected by both the coupling strength $\kappa L$ of the grating and by the position of the end mirror facets. The end mirror facets, which are randomly positioned relative to the grating, strongly affect which DFB mode lases. For some applications, especially gas sensing where absorption lines are sharp, it is essential to have good single-mode selection in order to position the emission frequency of the laser close to the target absorption feature. Better single-mode selection can be achieved by either depositing antireflection coatings on the end facets or by using a strongly overcoupled grating $(|\kappa L| \gg 1)$.

The variability of the threshold, slope efficiency, and output power among different lasers in the array is also caused by the end mirrors. To demonstrate the potential utility of DFB-QCL arrays, particularly their broad coverage range, we used them to perform infrared absorption spectroscopy on fluids.

Several development steps are left for future work. At present, the separate beams emerging from the individual lasers in the array are not combined into a single beam. Future work will include developing a tapered waveguide or other beam combining scheme to combine and collimate laser beams emitted from the QCL array into a single output. We also aim to increase the spectral coverage to several micrometers in wavelength. We hope to integrate our DFB-QCL array into a variety of spectroscopic devices, including a portable spectrometer operating in the mid-infrared.

\section{ACKNOWLEDGMENT}

The authors are grateful to D. Oakley, D. Chapman, and A. Napoleone of the Massachusetts Institute of Technology (MIT) Lincoln Laboratories for help in processing, and would also like to thank J. Faist of the Swiss Federal Institute of Technology (ETH) Zurich for valuable discussions. The Center for Nanoscale Systems (CNS) at Harvard University, which is a member of the National Nanotechnology Infrastructure Network (NNIN), is also gratefully acknowledged.

\section{REFERENCES}

[1] J. Faist, F. Capasso, D. L. Sivco, C. Sirtori, A. L. Hutchinson, and A. Y. Cho, "Quantum cascade laser," Science, vol. 264, pp. 553-556, 1994.

[2] F. Capasso, C. Gmachl, D. L. Sivco, and A. Y. Cho, "Quantum cascade lasers," Phys. Today, vol. 55, pp. 34-40, 2002.

[3] A. Lyakh, C. Pflügl, L. Diehl, Q. J. Wang, F. Capasso, X. J. Wang, J. Y. Fan, T. Tanbun-Ek, R. Maulini, A. Tsekoun, R. Go, C. Kumar, and N. Patel, "1.6 W high wall plug efficiency, continuous-wave room temperature quantum cascade laser emitting at $4.6 \mu \mathrm{m}$," Appl. Phys. Lett., vol. 92, pp. 111110-1-111110-3, 2008.

[4] Y. Bai, S. R. Darvish, S. Slivken, W. Zhang, A. Evans, J. Nguyen, and M. Razeghi, "Room temperature continuous wave operation of quantum cascade lasers with watt-level optical power," Appl. Phys. Lett., vol. 92, pp. 101105-1-101105-3, 2008.

[5] L. Diehl, D. Bour, S. Corzine, J. Zhu, G. Hofler, M. Lončar, M. Troccoli, and F. Capasso, "High-power quantum cascade lasers grown by low-pressure metalorganic vapor-phase epitaxy operating in continuous wave above $400 \mathrm{~K}$," Appl. Phys. Lett., vol. 88, pp. 201115-1-201115-3, 2006.

[6] R. Maulini, A. Mohan, M. Giovannini, J. Faist, and E. Gini, "External cavity quantum-cascade lasers tunable from 8.2 to $10.4 \mu \mathrm{m}$ using a gain element with a heterogeneous cascade," Appl. Phys. Lett., vol. 88, pp. 201113-1-201113-3, 2006 
[7] A. Kosterev and F. Tittel, "Chemical sensors based on quantum cascade lasers," IEEE J. Quantum Electron., vol. 38, no. 6, pp. 582-591, Jun. 2002.

[8] C. Gmachl, A. Straub, R. Colombelli, F. Capasso, D. L. Sivco, A. M. Sergent, and A. Y. Cho, "Single-mode, tunable distributed feedback and multiple wavelength quantum cascade lasers," IEEE J. Quantum Electron., vol. 38, no. 6, pp. 569-581, Jun. 2002.

[9] R. Maulini, M. Beck, J. Faist, and E. Gini, "Broadband tuning of external cavity bound-to-continuum quantum-cascade lasers," Appl. Phys. Lett., vol. 84, pp. 1659-1661, 2004.

[10] G. Wysocki, R. F. Curl, F. K. Tittel, R. Maulini, J. M. Bulliard, and J. Faist, "Widely tunable mode-hop free external cavity quantum cascade laser for high resolution spectroscopic applications," Appl. Phys. B, vol. 81, pp. 769-777, 2005.

[11] B. G. Lee, M. A. Belkin, R. Audet, J. MacArthur, L. Diehl, C. Pflugl, D. C. Oakley, D. Chapman, A. Napoleone, D. Bour, S. Corzine, G. Hofler, J. Faist, and F. Capasso, "Widely tunable single-mode quantum cascade laser source for mid-infrared spectroscopy," Appl. Phys. Lett., vol. 91, pp. 231101-1-231101-3, 2007.

[12] H. Kogelnik and C. V. Shank, "Coupled-wave theory of distributed feedback lasers," J. Appl. Phys., vol. 43, pp. 2327-2335, 1972.

[13] W. Streifer, R. Burnham, and D. Scifres, "Effect of external reflectors on longitudinal modes of distributed feedback lasers," IEEE J. Quantum Electron., vol. 11, no. 4, pp. 154-161, Apr. 1975.

[14] R. M. Williams, J. F. Kelly, J. S. Hartman, S. W. Sharpe, M. S. Taubman, J. L. Hall, F. Capasso, C. Gmachl, D. L. Sivco, J. N. Baillargeon, and A. Y. Cho, "Kilohertz linewidth from frequency stabilized mid-infrared quantum cascade lasers," Opt. Lett., vol. 24, pp. 1844-1846, 1999.

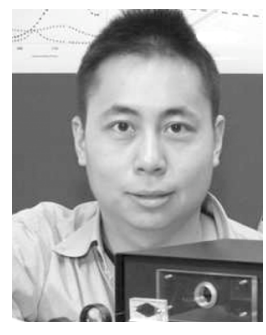

Benjamin G. Lee is a native of Toronto, Canada He received the B.S. degree in applied physics from Caltech, Pasadena, CA, in 2002. He is currently working toward the Ph.D. degree in applied physics at the School of Engineering and Applied Sciences, Harvard University, Cambridge, MA.

His current research interests include the development of novel quantum cascade laser devices for spectroscopy and chemical sensing, optoelectronics for solar energy harvesting, microfabrication, and nanotechnology.

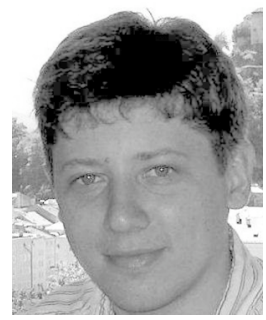

Mikhail A. Belkin received the B.S. degree in physics and mathematics from Moscow Institute of Physics and Technology, Moscow, Russia, in 1998, and the $\mathrm{Ph} . \mathrm{D}$. degree in physics from the University of California, Berkeley, in 2004.

From 2004 to 2008, he was with the School of Engineering and Applied Sciences, Harvard University, first as a Postdoctoral Fellow and later as a Research Associate. In August 2008, he has joined the Department of Electrical and Computer Engineering, University of Texas at Austin, Austin, as an Assistant Professor. His current research interests include the development of novel midinfrared and terahertz $(\mathrm{THz})$ quantum cascade laser designs, giant optical nonlinearities in semiconductor nanostructures, mid-infrared and THz photonic and plasmonic systems, and metamaterials in mid-infrared.

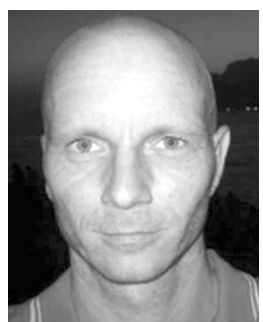

Christian Pflügl received the Diploma in physics from the University of Regensburg, Regensburg, Germany, in 2001, and the Ph.D. degree in electrical engineering from Vienna University of Technology, Vienna, Austria, in 2005.

From 2005 to 2006, he was a Postdoctoral Researcher at Vienna University of Technology, where he was engaged in research on quantum cascade lasers. In 2006, he joined Harvard University, Cambridge, MA. His current research interests include high-performance and single-mode quantum cascade lasers as well as the physics of these devices, nonlinear optics, mid-infrared plasmonics, microfabrication, and nanotechnology.

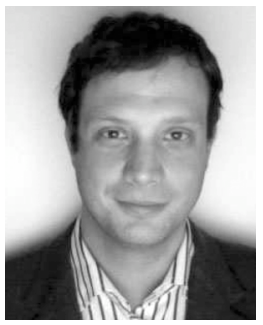

Laurent Diehl was born in Switzerland in 1976. He received the Ph.D. degree from the University of Neuchâtel, Neuchâtel, Switzerland, in 2003.

He was engaged in the development of p-type $\mathrm{Si} / \mathrm{SiGe}$ quantum cascade lasers. In 2003, he joined Harvard University, Cambridge, MA, as a Postdoctoral Fellow and later as a Research Associate. His current research interests include the development of high-power quantum cascade lasers grown by metal-organic chemical vapor deposition (MOCVD), as well as the physics of these devices, including their ultrafast dynamics.

Dr. Diehl was the corecipient of the Swiss Physical Society's International Business Machines Corporation (IBM) Award in 2001.

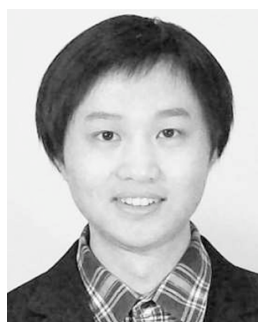

Haifei A. Zhang received the M.S. degree in electrical engineering from Zhejiang University, Hangzhou, China, in 2007. He is currently working toward the Ph.D. degree in applied physics at Harvard University, Cambridge, MA.

His current research interests include the development of quantum cascade laser devices and systems for spectroscopy, chemical sensing, and breath analysis as well as optoelectronics for energy technology.

Dr. Zhang is the Meritorious Winner of the Mathematical Contest in Modeling 2004 and the Intel Cup Embedded System Electronic Design Contest 2004.

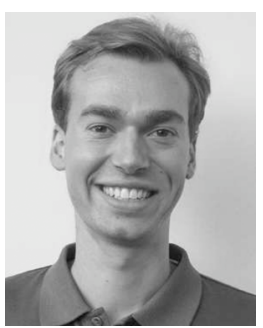

Ross M. Audet received the A.B. degree in engineering sciences and physics from Harvard University, Cambridge, MA, in 2007. He is currently working toward the Ph.D. degree in electrical engineering at Stanford University, Palo Alto, CA.

$\mathrm{He}$ was engaged in investigation of microcavity quantum cascade lasers at Harvard University. His current research interests include silicon-compatible optoelectronic devices and optical interconnects.

Mr. Audet is a Student Member of the Optical Society of America. He is the recipient of a Stanford

Graduate Fellowship.

Jim MacArthur received the B.S. degree in electrical engineering from Massachusetts Institute of Technology (MIT), Cambridge.

He is currently the Manager of the Electronic Instrument Design Laboratory, Department of Physics, Harvard University, Cambridge.

David P. Bour (S'84-M'85-SM'97-F'00) was born in Pittsburgh, PA. He received the B.S. degree in physics from Massachusetts Institute of Technology, Cambridge, in 1983, and the Ph.D. degree in electrical engineering from Cornell University, Ithaca, NY, in 1988.

$\mathrm{He}$ is currently the Chief Scientist at BridgeLux, Inc., Sunnyvale, CA, which is a high-brightness LED manufacturer. He is also with Photodigm, Inc., Richardson, TX. He was the Engineering Director with the New Business and New Products Group of Applied Materials, where he was engaged in the development of metal-organic chemical vapor deposition growth technology for compound semiconductors. From 1987 to 1991, he was a Member of the Technical Staff with Sarnoff Corporation, Princeton, NJ. From 1991 to 1999, he was a Principal Scientist with the Electronic Materials Laboratory, Xerox Palo Alto Research Center, Palo Alto, CA, fabricating nitride blue laser diodes and phosphide red laser diodes for laser printing. From 1999 to 2005, he was an Agilent Fellow with the Photonics and Electronics Research Laboratory, Agilent Laboratories, where he was engaged in the development of semiconductor lasers for electronics, compact disk (CD), and digital video disk (DVD) data storage, communications, and projection displays. He has authored or coauthored more than 200 articles and several book chapters. $\mathrm{He}$ is the holder of 60 U.S. patents. 
Scott W. Corzine was born in Nashville, TN, in 1963. He received the B.S., M.S., and Ph.D. degrees from the Department of Electrical and Computer Engineering, University of California at Santa Barbara, Santa Barbara, in 1985, 1987, and 1993, respectively.

He was at Agilent Laboratories, Palo Alto, CA, where he was engaged in the design and fabrication of various optoelectronic devices for ten years. He is currently with Infinera, Sunnyvale, CA. He has coauthored a graduate-level textbook on semiconductor lasers and has also contributed a chapter to a book on quantum well lasers.

Gloria E. Höfler received the B.S., M.S., and Ph.D. degrees in electrical engineering from the University of Illinois at Urbana-Champaign, Urbana, in 1986, 1989, and 1993, respectively.

From 1992 to 1994, she was with the 3M Photonics Laboratory, where she was involved in the development of II-VI blue-green lasers. From 1994 to 2001, she held various engineering and managerial positions with Hewlett-Packard and Lumileds in the area of III-V opto-electronics ranging from device design and fabrication to packaging. In 2001, she joined Agilent Technologies, Palo Alto, CA, where she was involved with the development of high-speed components for datacom, metro networks, and sensing applications. She is currently with Argos Technologies, Santa Clara, CA. She has authored or coauthored over 40 peer-reviewed publications. She holds several patents.

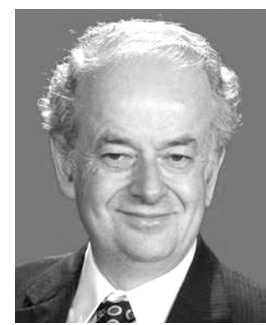

Federico Capasso (M'79-SM'85-F-87) received the Dr. Phys. degree (summa cum laude) from the University of Rome, Rome, Italy, in 1973.

$\mathrm{He}$ is currently the Robert Wallace of Applied Physics and Vinton Hayes Senior Research Fellow in electrical engineering at Harvard University, Cambridge, MA. From 1974 to 1976, he was with Fondazione Ugo Bordoni as a Researcher. In 1976, he joined Bell Laboratories, where he was a Member of the Technical Staff (1977-1986), the Department Head (1986-2000), and the Vice President for Physical Research (2000-2002). In 1997, he was made a Bell Laboratories Fellow for his scientific contributions. He has been engaged in basic and applied research on the design of new artificial materials and devices, using an approach he pioneered known as band structure engineering, in investigations on quantum electrodynamical phenomena such as the Casimir effect and on surface plasmon photonics. He is a coinventor of the quantum cascade laser. His recent achievements include the invention and demonstration of low-divergence semiconductor lasers with plasmonic collimators, plasmonic laser antennas, and the first measurement of repulsive Casimir forces. He has authored or coauthored more than 300 papers, edited four volumes, and holds over 55 U.S. patents.

Dr. Capasso is a member of the National Academy of Sciences and the National Academy of Engineering, and an Honorary Member of the Franklin Institute. He was the recipient of the King Faisal International Prize for Science, the IEEE Edison Medal, the Arthur Schawlow Prize in Laser Science, the Wetherill Medal of the Franklin Institute, the Robert Wood Prize of the Optical Society of America (OSA), the William Streifer Award of the Laser and Electro-Optic Society (IEEE), the Rank Prize in Optoelectronics (U.K.), the IEEE David Sarnoff Award in Electronics, the Duddell Medal of the Institute of Physics (U.K.), the Willis Lamb Medal for Laser Science and Quantum Optics, the Materials Research Society Medal, the "Vinci of Excellence" Prize (France), the Welker Memorial Medal (Germany), the New York Academy of Sciences Award, the Newcomb Cleveland Prize of the American Association for the Advancement of Science, and the National Aeronautics and Space Administration (NASA) Achievement Award. He is a Fellow of the Optical Society of America, the American Physical Society, the International Society for Optical Engineering (SPIE), the American Association for the Advancement of Science (AAAS), and the American Academy of Arts and Sciences. 